Article

\title{
Ascorbyl Tetraisopalmitate Inclusion into Y-Cyclodextrin and Mesoporous SBA-15: Preparation, Characterization and In Vitro Release Study
}

\author{
Maria Bastianini * (D), Michele Sisani (D) and Annarita Petracci \\ Prolabin \& Tefarm S.r.l., R\&D Department, Via dell'Acciaio 9, 06134 Perugia, Ponte Felcino, Italy; \\ michele.sisani@prolabintefarm.com (M.S.); annarita.petracci@prolabintefarm.com (A.P.) \\ * Correspondence: maria.bastianini@prolabintefarm.com; Tel.: +39-328-7142344
}

Received: 30 May 2017; Accepted: 29 June 2017; Published: 1 July 2017

\begin{abstract}
Ascorbic acid or vitamin C is a strong antioxidant widely used in cosmetic and food fields. This vitamin is very unstable and rapidly undergoes degradation. In order to solve this problem and to obtain a stable ascorbic acid, Nikkol Group has developed ascorbyltetraisopalmitate (VC-IP). This raw material is an oil phase, already well-known and employed in the cosmetic market. The objective of this study is to obtain VC-IP in micro-powder form, in order to produce a new raw material that is easily dispersible in oil and water phases and useful for make-up and color cosmetic applications. Various types of drug carriers were studied and considered in order to support VC-IP and obtain the conversion in powder. Y-cyclodextrin and mesoporous silica SBA-15 were chosen as the best candidates. A white powder of supported VC-IP was obtained with each carrier (VC-IP@cyclodextrin, VC-IP@SBA-15). The systems underwent physicochemical characterization and in vitro release tests were carried out. Based on the conducted study, it can be concluded that by supporting VC-IP on Y-cyclodextrin and SBA-15, it is feasible to obtain a new raw material in powder form. The two carriers possess different release profiles, adding the possibility to finely tune the release of the active component in smart formulations.
\end{abstract}

Keywords: ascorbic acid; hybrid materials; controlled release

\section{Introduction}

Vitamin $C$ is a natural anti-aging and whitening bioactive ingredient that has been widely used in cosmetic and dermatological products thanks to its favorable effects on the skin [1], which include radicals scavenging, collagen biosynthesis, and the suppression melanin formation [2-5]. The strong antioxidant properties and the ability to prevent skin aging make ascorbic acid, also known as vitamin $\mathrm{C}$, one of the most interesting active ingredients for the cosmetic industry. However, its employment in products for topical use (skin care, deodorants, hair dyes, etc.) is severely limited because of its instability. In fact, ascorbic acid tends to rapidly degrade due to an oxidation process, causing a consequent decline in the title and then the loss of activity [6]. Various strategies have been pursued in order to avoid this problem, for instance the exclusion of oxygen or the use of air impermeable packaging, and the use of carrier and release systems [7] that protect the vitamin $C$ from oxygen, water, and other components that may be included in the cosmetic formulation. A very stable form of ascorbic acid is vitamin C ascorbyl tetraisopalmitate (VC-IP). VC-IP (Figure 1) is stable at high temperatures and has good solubility in oils. It exhibits excellent percutaneous absorption and effectively converts into free vitamin $C$ in the skin to perform various physiological functions [8,9]. VC-IP is approved as a quasi-drug active in Japan (at $3 \mathrm{wt} \%$ ). It is also registered in Korea as a functional ingredient for skin lightening at $2 \mathrm{wt} \%$ concentration. 


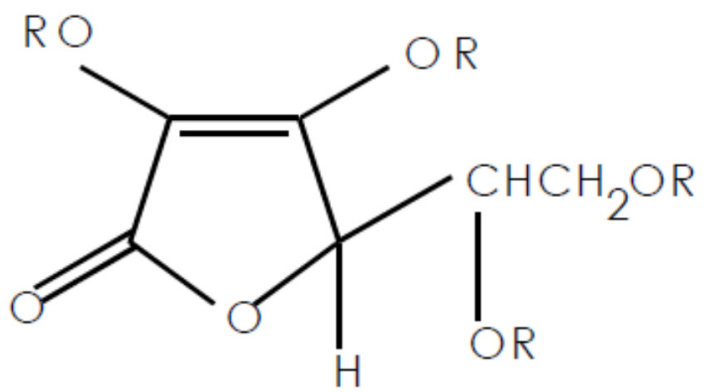

Figure 1. Chemical structure of VC-IP, in which R is an isopalmitate group.

VC-IP is an oil-soluble raw material in the form of a colorless to pale yellow liquid, and for this reason some cosmetic applications are precluded. In fact, it is difficult to dissolve VC-IP in water or use it in compact formulations for make-up and color cosmetics. Therefore, it could be very interesting and strategic to find a formulation of VC-IP in powder form in order to expand the field of application of this raw material. The idea of this study is to include VC-IP in a solid matrix in order to develop a new cosmetic raw material in micro-powder form. This strategy could allow the easy dispersion of the active ingredient both in water and oil phases of semisolid formulations, as well as allow its direct use in compacts and other solid formulations for color cosmetic applications. The confinement of the active ingredient further decreases the oxidative degradation of VC-IP, because even in case of formation of by-product radicals, it is likely that the radicals recombine, bringing the molecule to the initial state. Thus, this strategy can greatly improve the stability of VC-IP. Moreover, the use of a carrier to vehiculate VC-IP can successfully improve the release of the active ingredient, producing a formulation with modified and prolonged delivery. Taking into account that VC-IP is a neutral molecule among the different types of solid drug carriers [10-12], Cyclodextrin and mesoporous SBA-15 have been chosen as the best candidates for the purpose of this work. Cyclodextrin are cyclic $(\alpha-1,4)$-linkedoligosaccharides of $\alpha$-D-glucopyranose, containing a relatively hydrophobic central cavity and hydrophilic outer surface. During the past two decades, cyclodextrin and their derivatives have attracted considerable interest in the pharmaceutical field because of their potential ability to form complexes with a variety of drug molecules. Cyclodextrin are used to increase the solubility of water-insoluble drugs through the formation of inclusion complexes. The hydrophobic cavity of cyclodextrin is capable of trapping a variety of molecules to produce inclusion complexes [13]. Three types of cyclodextrin that are most known and widely used and are $\alpha, \beta$, and Y. The difference between them is the dimension of the cavity [13]. Y-cyclodextrin was chosen because it offers the biggest cavity, which was considered adept to host the active ingredient of VC-IP, which is an ester in which ascorbic acid is covalent linked to four palmitates, (C 16), with a big steric hindrance.

SBA-15 (Santa Barbara Amorphous type material) is an ordered mesoporous silicate. Due to its unique properties, it appears to be an ideal solution for the encapsulation of pharmaceutical drugs, proteins, and other biogenic molecules. In addition, it is very effective in terms of the enhancement of active dissolution [14].

\section{Materials and Methods}

\subsection{Chemicals and Solvent}

SBA-15 was purchased by Sigma-Aldrich (Saint Louis, Missouri, USA). Y-cyclodextrin was purchased from Nordmann Rassmann $\mathrm{GmbH}$ (Hamburg, Germany). Ethanol was provided by Applichem Panreac (Darmstadt, Germany) and VC-IP by Nikkol Group (Tokyo, Japan). The solvent and materials were of reagent grade and were used without further purification. 


\subsection{VC-IP@cyclodextrin Preparation}

For the preparation of VC-IP@cyclodextrin, $459 \mathrm{mg}$ of VC-IP was dissolved in $8 \mathrm{~mL}$ of ethanol in order to have a $0.05 \mathrm{M}$ solution. Then, $1 \mathrm{~g}$ of Y-cyclodextrin was added to the $8 \mathrm{~mL}$ of VC-IP solution. The final dispersion was stirred for $24 \mathrm{~h}$ at room temperature, protected from light and air. The solvent was then eliminated using a rotavapor, and a dry white powder was obtained. In order to have an exact reference for X-ray powder diffraction analysis, the experiment was repeated without VC-IP. Therefore, pure cyclodextrin was dissolved in ethanol, stirred for $24 \mathrm{~h}$, and the solvent was evaporated using a rotavapor.

\subsection{VC-IP@SBA-15 Preparation}

For the preparation of VC-IP@SBA-15, $400 \mathrm{mg}$ of VC-IP was dissolved in $14 \mathrm{~mL}$ of ethanol in order to have a $0.025 \mathrm{M}$ solution. Then, $1 \mathrm{~g}$ of SBA- 15 was added to the $14 \mathrm{~mL}$ of VC-IP solution. The SBA/VC-IP weight ratio was 2.5 and the final solution was stirred for $24 \mathrm{~h}$ at room temperature, protected from light and air. Then the solvent was eliminated using a rotavapor, and a dry white powder was obtained.

\subsection{Sample Characterization}

\subsubsection{X-ray Powder Diffraction}

The samples were characterized by X-ray powder diffraction spectrometry (XRPD). XRPD patterns were recorded with a Philips X'PERT PRO MPD diffractometer operating at $40 \mathrm{kV}$ and $40 \mathrm{~mA}$, with a step size of 0.0170 degree and a step scan rate of $20 \mathrm{~s}$, using $\mathrm{Cu}$ Ka radiation and an $\mathrm{X}^{\prime}$ Celerator detector.

\subsubsection{Thermal Analysis}

Thermogravimetric (TGA) and differential scanning calorimetry (DSC) analyses were carried out with an STD Q600 thermal analyzer TA Instrument, in air flow with a heating rate of $10^{\circ} \mathrm{C} / \mathrm{min}$.

\subsubsection{Field Emission Scanning Electron Microscopy}

The morphology of the samples was investigated with a FEG LEO 1525 scanning electron microscope (FE-SEM). FE-SEM micrographs were collected by depositing the samples on a stub holder and after a sputter coating with chromium for $20 \mathrm{~s}$.

\subsection{Extraction and HPLC Method for the Determination of VC-IP Content in the Hybrid Material}

The amount of VC-IP in the samples was determined using a High-Performance Liquid Chromatography (HPLC) method under the following conditions:

Column: Zorbax eclipse XDB $4.6 \times 150 \mathrm{~mm} 5$ micron

Mobile phase: methanol/isopropanol 25/75, isocratic

Column temperature: $30^{\circ} \mathrm{C}$

Sample volume: $20 \mu \mathrm{L}$

Flux: $1 \mathrm{~mL} / \mathrm{min}$

Detector: Variable Wavelength Detector $(\lambda=222 \mathrm{~nm})$

Retention time: $5.9 \mathrm{~min}$

Using these experimental conditions, a suitable calibration was carried out by dissolving known amounts of pure VC-IP in proper volumes of a methanol/isopropanol 50/50 solution. Five VC-IP standards and a blank sample were then prepared and analyzed by HPLC in order to obtain the calibration curve. The linearity obtained was satisfactory and presented a correlation coefficient of 0.9975. Accuracy, precision, and sensitivity of the method are reported in the reference article of Almeida et al. [15] where the method was developed. 
Samples of analysis were prepared by treating the VC-IP hybrids under the following conditions:

(1) A known amount of sample was dispersed by stirring in a proper volume of a methanol/isopropanol 50:50 solution.

(2) The dispersion was treated for $30 \mathrm{~min}$ in an ultrasound bath.

(3) Finally, the dispersion was filtered using a $0.20-\mu \mathrm{m}$ pore size filter.

VC-IP determinations on the extracted volume were repeated in triplicate and the content of the solid was reported as an average. The test was repeated after one year of storage at $25^{\circ} \mathrm{C}$ and $50 \%$ of relative humidity. Both the samples showed perfect stability after one year of aging.

\subsection{Franz Cell Release}

VC-IP release studies of pure VC-IP, VC-IP@cyclodextrin, and VC-IP@SBA-15 were performed using a Franz Diffusion Cell (PermeGear, Inc., Bethlehem, Palestine, $20 \mathrm{~mm}$ in diameter) equilibrated at $32{ }^{\circ} \mathrm{C}$ by an external circle bath. The Franz Cell is composed of a donor chamber and a receiver chamber with a volume of $15 \mathrm{~mL}$. These two parts were separated by a circular cellulose membrane (Filter paper Whatman 41, 20-25 mm, Whatman GmbH, Dassel, Germany). Ethanol was chosen as donor and acceptor solvent in order to ensure the sink conditions. Pure VC-IP (100 mg), VC-IP@cyclodextrin (335 mg, corresponding to $100 \mathrm{mg}$ of pure VC-IP) and VC-IP@SBA-15 (347 mg, corresponding to $100 \mathrm{mg}$ of pure VC-IP) were inserted in the donor chambers. Aliquots $(0.40 \mathrm{~mL})$ of the acceptor fluid were collected at predetermined time intervals ( $5 \mathrm{~min}, 10 \mathrm{~min}, 20 \mathrm{~min}, 30 \mathrm{~min}, 60 \mathrm{~min}, 120 \mathrm{~min}, 180 \mathrm{~min}$, $240 \mathrm{~min}, 300 \mathrm{~min}, 360 \mathrm{~min}$, and $420 \mathrm{~min}$ ) and immediately replaced by the same volume of ethanol equilibrated at $32{ }^{\circ} \mathrm{C}$. The collected ethanol was properly diluted. The VC-IP released was detected by UV spectrophotometry at the wavelength of maximum absorption of VC-IP in the solvent $(\lambda=223 \mathrm{~nm})$ by using an UV-visible double-beam spectrophotometer (Jasco V-750, Easton, MD, USA). Tests were conducted in triplicate and the results were reported as an average.

\section{Results and Discussion}

\subsection{VC-IP@cyclodextrin Characterization}

The inclusion method produced a homogeneous white powder sample. In order to verify the effective formation of cyclodextrin/VC-IP complex, XRPD analysis on the sample was carried out (Figure 2). The spectrum recorded on the gamma-cyclodextrin after the contact with VC-IP was significantly different from that of the pristine cyclodextrin matrix treated in the same way. In particular, the spectrum of the VC-IP inclusion compound showed an amorphous structure suggesting the formation of gamma-cyclodextrin/VC-IP complex, as indicated in literature [16,17]. A further evidence of the effective VC-IP vehiculation was obtained by DSC analysis. In Figure 3, the curves of the pristine gamma-cyclodextrin, the pure VC-IP, and the cyclodextrin/VC-IP complex are reported. In the DSC curve of the cyclodextrin/VC-IP complex the peak of degradation of the VC-IP was absent, while the same peak at about $350{ }^{\circ} \mathrm{C}$ is clearly visible in the curve of the pure active ingredient [18]. In fact, in the DSC curve of the complex, only the signals of the cyclodextrin were present, suggesting a good interaction between the matrix and the VC-IP [19], which probably enhances the thermal stability of the active ingredient. 


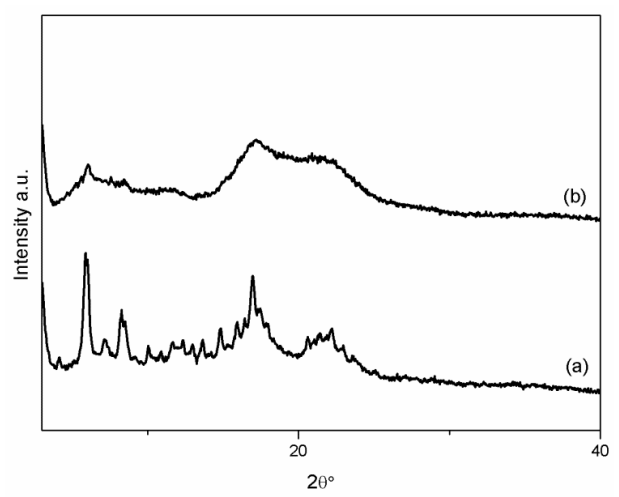

Figure 2. X-ray diffraction patterns of the pure ethanol-treated cyclodextrin (a) and of the VC-IP@cyclodextrin sample (b).

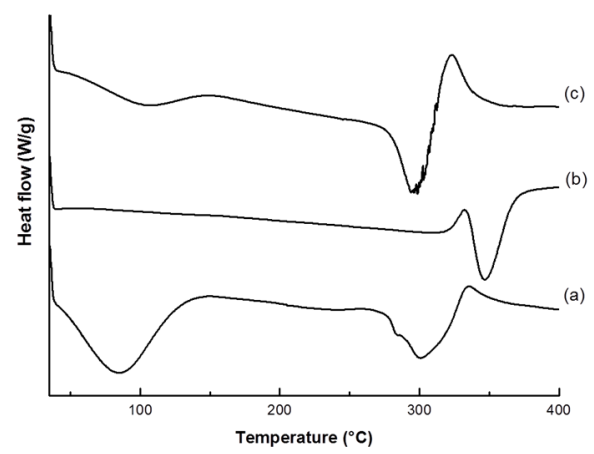

Figure 3. Differential scanning calorimetry analysis of the pure cyclodextrin (a), of the VC-IP (b), and of the sample VC-IP@cyclodextrin (c).

In order to determine the exact content of VC-IP in the sample, the active ingredient was extracted from the hybrid by sonication treatment in ethanol and the obtained solution was analyzed by HPLC [15]. The experimental VC-IP content was $29.8 \mathrm{wt} \%$. After one year of storage, the content was re-determined and confirmed, showing that the VC-IP in the complex was stable.

The powder sample was analyzed with FE-SEM in order to determine the dimensions and morphology of the particles. Figure 4 shows a micrograph of VC-IP@cyclodextrin.

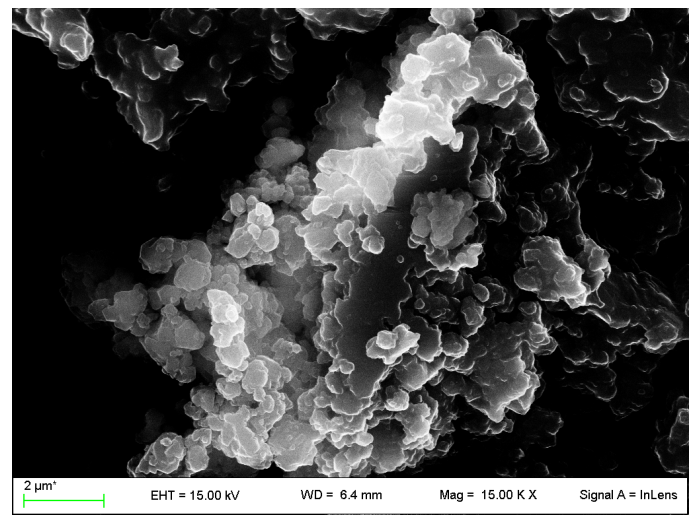

Figure 4. FE-SEM micrographs of VC-IP@cyclodextrin.

In the micrographs, particles are present with irregular morphology and with dimensions of $300-500 \mathrm{~nm}$, that are fused in a micrometric aggregate. 


\subsection{VC-IP@SBA-15 Characterization}

The white homogeneous powder obtained was characterized by TGA, DSC, HPLC, and FE-SEM.

The TGA analysis carried out on the SBA-15 before and after the contact with VC-IP showed a large difference in the weight loss (Figure 5). In particular, the pristine SBA-15 showed a weight loss of 5.5\%, whereas the VC-IP@SBA-15 sample showed a weight loss of $36.3 \%$. This difference is due to the loss of residual ethanol and to the thermal decomposition of the VC-IP. The VC-IP loading observed by TGA analysis is about $28 \mathrm{wt} \%$. The active in the sample was extracted as indicated in the experimental part, and the obtained solution was analyzed by the HPLC method to determine the exact VC-IP loading. The experimental active content was $28.8 \mathrm{wt} \%$, a value very close to the one obtained by TGA analysis. Also in this case, after one year of storage in controlled conditions, the VC-IP content was confirmed. Both the samples showed a high stability and, as evidenced by the HPLC analysis, no ascorbic acid degradation was detected even after 12 months of aging.

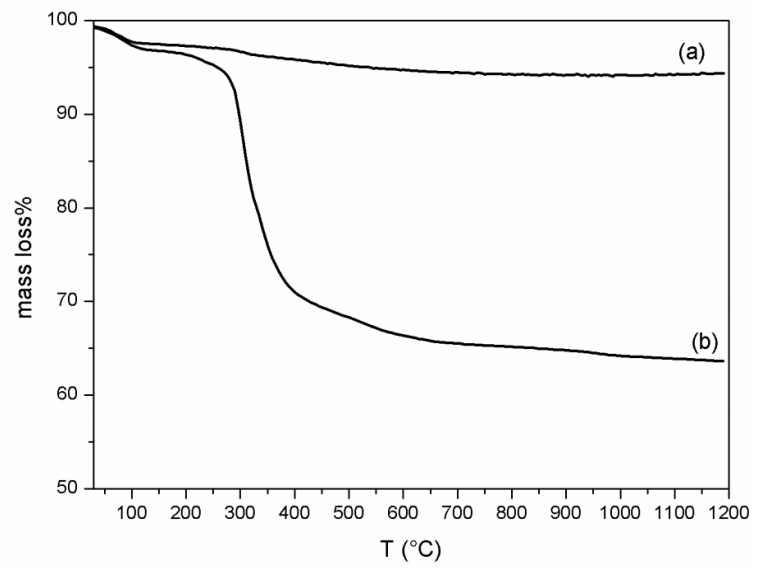

Figure 5. TGA of SBA-15 (a) and VC-IP@SBA-15 (b).

The comparison of the DSC curves registered on the SBA-15, VC-IP, and VC-IP@SBA-15 samples showed the disappearance of the thermal degradation peak of the VC-IP in the VC-IP@SBA-15 sample, as observed for the gamma-cyclodextrin material (Figure 6).

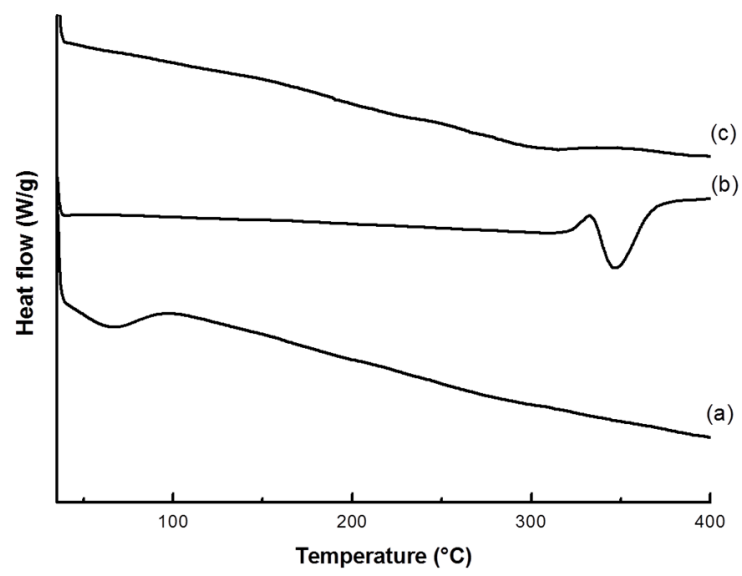

Figure 6. DSC curves of SBA-15 (a), VC-IP (b), and VC-IP@SBA-15 (c).

Figure 7 reports the FE-SEM micrograph of the sample. The sample shows a regular and tubular morphology. 


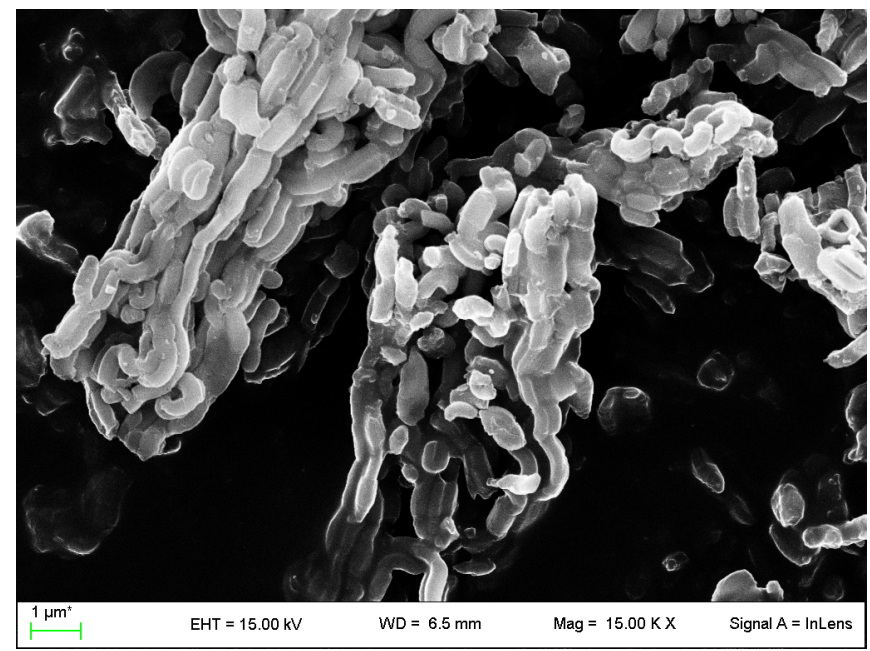

Figure 7. FE-SEM micrographs of VC-IP@SBA-15.

\subsection{In Vitro Release Test}

The in vitro release performance of the included VC-IP was assessed using ethanol as a solvent in order to ensure the sink conditions. In fact, VC-IP in water or in water/ethanol mixture is not soluble; preliminary solubility tests were carried out, and it was found that when adding VC-IP to water and to a water/ethanol mixture (30:70, 20:80, and 10:90 were tested), turbid emulsions were always obtained. All release experiments showed good reproducibility. The release profiles of the VC-IP from SBA-15 and Y-cyclodextrin compared to the dissolution of pure VC-IP are shown in Figure 8. The results are expressed as \% (mass \% referring to the total active component in the donor cell) of the released VC-IP in the dissolution solvent referred to the total VC-IP content in the donor cells.

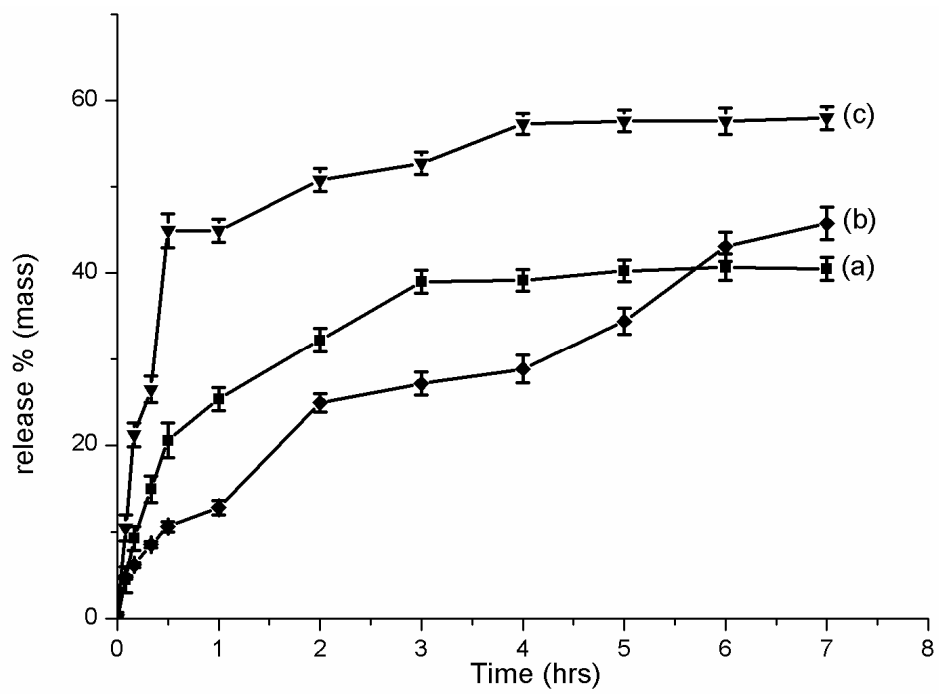

Figure 8. Release profile of pure VC-IP (a), VC-IP@SBA-15 (b), VC-IP@cyclodextrin (c).

For the pure VC-IP, the maximum release is about $40 \%$. The initial release is quite fast and the plateau is reached at $3 \mathrm{~h}$.

In the case of VC-IP@SBA-15, the release of the active ingredient from the mesoporous silica is more gradual. From the release curve, it is clearly visible that the content of VC-IP in the acceptor fluid gradually increases over time. The maximum amount released is higher than the one obtained 
with the pure VC-IP. The release curve shows that this sample could be used for the preparation of formulations with a prolonged release of the active ingredient.

VC-IP@cyclodextrin shows a fast release of VC-IP even after $5 \mathrm{~min}$, and it reaches values of release considerably higher that the pure active ingredient. After $7 \mathrm{~h}$, the release from the cyclodextrin matrix is $58 \%$ while the pure VC-IP shows only $40 \%$ release. It is well known that cyclodextrins act as true carriers by keeping the hydrophobic drug molecules in solution. Inclusion in cyclodextrins exerts a profound effect on the physicochemical properties of guest molecules as they are temporarily locked or caged within the host cavity, giving rise to beneficial modifications of guest molecules, such as solubility enhancement, which are not achievable otherwise [20]. The molecule is directly released from the complex and does not need to be solubilized, giving an increase of "apparent solubility" of the active ingredient [21]. The experimental data confirmed that the structure of cyclodextrin could be successfully employed to obtain effective fast release formulations.

\section{Conclusions}

This ascorbyl tetraisopalmitate in micro-powder form was successfully obtained using SBA-15 and Y-cyclodextrin as solid supports. The VC-IP powder was white and showed a very high stability, opening new possibilities for the application of this active ingredient. VC-IP@cyclodextrin and VC-IP@SBA15 showed different release profiles in the in vitro tests, and both of them can be used to improve the VC-IP release in different ways. VC-IP@cyclodextrin can be used for a fast and high release, while VC-IP@SBA15 could be useful for the development of formulations with a prolonged release. They allow for the control of the active release, thus increasing the efficacy of the active ingredient.

VC-IP in powder form could be a real innovation in compacts and other solid formulations for colour cosmetic applications with whitening and anti-age effects. Currently, the use of this active component in compact formulations is precluded due to its oil form. This new raw material could be dispersed in water and oil phases of semisolid formulations, but more studies will be conducted in order to ensure the stability of the samples in these conditions and their ability to be good carriers, not only in powder form. VC-IP in powder form could greatly increase the use of this active ingredient in the cosmetic market, because it allows for the safe and effective application of the vitamin $C$ derivative in many fields.

Acknowledgments: The authors would like to thank Nikkol Group for the scientific collaboration.

Author Contributions: All the authors has contributed equally to the development of the article.

Conflicts of Interest: The authors declare no conflict of interest.

\section{References}

1. Maia, C.P.; Mara, S.G. Ascorbic acid and its derivatives in cosmetic formulations. Cosmet. Toilet. 2000, 115, 59-62.

2. Darr, D.; Combs, S.; Dunston, S.; Manning, T.; Pinnell, S. Topical vitamin C protects porcine skin from ultraviolet radiation-induced damage. Br. J. Dermatol. 1992, 127, 247-253. [CrossRef] [PubMed]

3. Bagchi, D.; Garg, A.; Krohn, R.; Bagchi, M.; Tran, M.X.; Stohs, S.J. Oxygen free radical scavenging abilities of vitamins $\mathrm{C}$ and $\mathrm{E}$, and a grape seed proanthocyanidin extract in vitro. Res. Commun. Mol. Pathol. Pharmacol. 1997, 95, 179-189. [PubMed]

4. Linster, C.L.; Van Schaftingen, E. Vitamin C Biosynthesis, recycling and degradation in mammals. Febs J. 2007, 274, 1-22. [CrossRef] [PubMed]

5. Patricia, K.; Farris, M.D. Topical Vitamin C: A Useful Agent for Treating Photoaging and Other Dermatologic Conditions. Dermatol. Surg. 2005, 31, 814-818.

6. Sheraz, M.A.; Ahmed, S.; Ahmad, I.; Shaikh, R.H.; Vaid, F.H.M.; Iqbal, K. Formulation and Stability of Ascorbic Acid in Topical Preparations. Syst. Rev. Pharm. 2011, 2, 86-90. [CrossRef] 
7. Gu, C.; Hu, C.; Ma, C.; Fang, Q.; Xing, T.; Xia, Q. Development and characterization of solid lipid microparticles containing vitamin C for topical and cosmetic use. Eur. J. Lipid Sci. Technol. 2016, 118, 1093-1103. [CrossRef]

8. Ochiai, Y.; Kaburagi, S.; Obayashi, K.; Ujiie, N.; Hashimoto, S.; Okano, Y.; Masaki, H.; Ichihashi, M.; Sakurai, H. A new lipophilic pro-vitamin C, tetra isopalmitoyl ascorbic acid (VC-IP), prevents UV-induced skin pigmentation through its anti-oxidative properties. J. Dermatol. Sci. 2006, 44, 37-44. [CrossRef] [PubMed]

9. Maia Campos, P.M.B.G.; Gianeti, M.D.; Camargo, F.B., Jr.; Gaspar, L.R. Application of tetra-isopalmitoyl ascorbic acid in cosmetic formulations: Stability studies and in vivo efficacy. Eur. J. Pharm. Biopharm. 2012, 82, 580-586. [CrossRef] [PubMed]

10. Patravale, V.B.; Mandawgade, S.D. Novel cosmetic delivery systems: An application update. Int. J. Cosmet. Sci. 2008, 30, 19-33. [CrossRef] [PubMed]

11. Wang, S. Ordered mesoporous materials for drug delivery. Microporous Mesoporous Mater. 2009, 117, 1-9. [CrossRef]

12. Casanova, F.; Santos, L. Encapsulation of cosmetic active ingredients for topical application-A review. J. Microencapsul. 2016, 33, 1-17. [CrossRef] [PubMed]

13. Szejtli, J. Introduction and General Overview of Cyclodextrin Chemistry. Chem. Rev. 1998, 98, $1743-1753$. [CrossRef] [PubMed]

14. Vallet-Regi, M.; Balas, F.; Arcos, D. Mesoporous Materials for Drug Delivery. Angew. Chem. Int. Ed. Engl. 2007, 46, 7548-7558. [CrossRef] [PubMed]

15. Almeida, M.M.; Alves, J.M.P.; Patto, D.C.S.; Lima, C.R.R.C.; Quenca-Guillen, J.S.; Santoro, M.I.R.M.; Kedor-Hackmann, E.R.M. Determination of tocopheryl acetate and ascorbyl tetraisopalmitate in cosmetic formulations by HPLC. Int. J. Cosmet. Sci. 2009, 31, 445-450. [CrossRef] [PubMed]

16. Sinha, R.; Anitha, R.; Ghosh, S.; Nanda, A.; Kumria, R. Complexation of celecoxib with $\beta$-cyclodextrin: Characterization of the interaction in solution and in solid state. J. Pharm. Sci. 2005, 94, 676-687. [CrossRef] [PubMed]

17. Lee, P.S.; Han, Y.-H.; Song, T.W.; Sung, J.H.; Kwon, O.-S.; Song, S.; Chung, Y.-B. Physicochemical characteristics and bioavailability of a novel intestinal metabolite of ginseng saponin (IH901) complexed with gamma-cyclodextrin. Int. J. Pharm. 2006, 316, 29-36. [CrossRef] [PubMed]

18. Mandelli de Almeida, M.; Ribeiro de Castro Lima, C.R.; Quenca-Guillen, J.S.; Filho, E.M.; Mercuri, L.P.; Rocha Miritello Santoro, M.I.; Kedor-Hackmann, E.R.M. Stability evaluation of tocopheryl acetate and ascorbyl tetraisopalmitate in isolation and incorporated in cosmetic formulations using thermal analysis. Braz. J. Pharm. Sci. 2010, 46, 129-134. [CrossRef]

19. Singh, R.; Bharti, N.; Madan, J.; Hiremath, S.N. Characterization of Cyclodextrin Inclusion Complexes-A Review. J. Pharm. Sci. Technol. 2010, 2, 171-183.

20. Martin Del Valle, E.M. Cyclodextrins and their uses: A review. Process Biochem. 2004, 39, $1033-1046$. [CrossRef]

21. Davis, E.M.; Brewster, E.M. Cyclodextrin-based pharmaceutics: Past, present and future. Nat. Rev. Drug Discov. 2004, 3, 1023-1035. [CrossRef] [PubMed]

(C) 2017 by the authors. Licensee MDPI, Basel, Switzerland. This article is an open access article distributed under the terms and conditions of the Creative Commons Attribution (CC BY) license (http://creativecommons.org/licenses/by/4.0/). 